\title{
A model to facilitate the development of an appropriate risk assessment methodology and instrument for crowd safety at outdoor music festivals
}

\author{
A. Raineri \\ School of Human, Health and Social Sciences, CQ University, Australia
}

\begin{abstract}
Attendance at outdoor music festivals is associated with an increased risk of injury and death. A considerable proportion of crowd-related risks are attributed to irrational and high-risk behaviour by patrons, especially in the general admission, or standing room only areas in front of stages, or 'mosh pits'. Risk assessments for music festivals and mass gatherings generally tend to deal with the traditional hazards and risks found at most workplaces, without taking into account the dynamics of the crowd or those factors that influence its behaviour. Influences on crowd behaviour are little understood and generally ignored, leaving a significant source of risk at this type of event unaccounted for. A comprehensive approach to crowd safety assessment, design and management needs to integrate both psychological and engineering frames of reference. This paper outlines a model that can be used as the basis for developing a contextualised methodology and instrument for assessing crowd related risks at outdoor music festivals.
\end{abstract}

Keywords: outdoor music festivals, crowd safety assessment, crowd behaviour.

\section{Introduction}

Outdoor music festivals are increasingly common events on the summer entertainment landscape for youth in many countries around the world. Evidence indicates that attendance is associated with an increased risk of injury and, in extreme cases, death $[1,2]$. A considerable proportion of crowd-related risks are attributed to irrational and high-risk behaviour by patrons in the general 
admission or standing room only areas in front of stages, or 'mosh pits' [3]. Ingress and egress are equally, if not more, problematic and the likelihood of problems in crowds is increased by disruptions to movement flows and reactions to perceived risk or competitive rushes.

While there is considerable endorsement in the literature for a risk management approach, its application to crowd safety is rare. Risk assessments for music festivals and mass gatherings generally tend to deal with the traditional hazards and risks found at most workplaces without taking into account the dynamics of the crowd or those factors that influence its behaviour.

Insufficient attention to the way that people behave in a crowd and the relationship between behaviour and systems design are major factors in crowd disasters [4, 5]. A comprehensive approach to crowd safety assessment, design and management needs to integrate both psychological and engineering frames of reference [6-8].

In order to be able to develop an appropriate and effective risk assessment method and tool, it is proposed to initially develop a model that identifies and maps relevant characteristics in an organised and systematic manner and shows the effect of these on processes that transform individual into collective behaviour. While the model will commence with factors already outlined by commentators, further research will need to be undertaken to identify any additional relevant factors. Because a model is a conceptual representation whose purpose is to explain and predict observed phenomena, such an approach provides a robust platform on which to build the projected methodology and instrument.

\section{Identifying a typology of domains}

The 'mass gathering' literature demonstrates that there are a number of key characteristics of an event that impact on patrons' health and safety. Arbon [9] has organised these characteristics into three primary domains: the psychosocial, environmental and bio-medical domains, as outlined in Figure 1. Each domain has set characteristics which help to explain the impact on the mass gathering event.

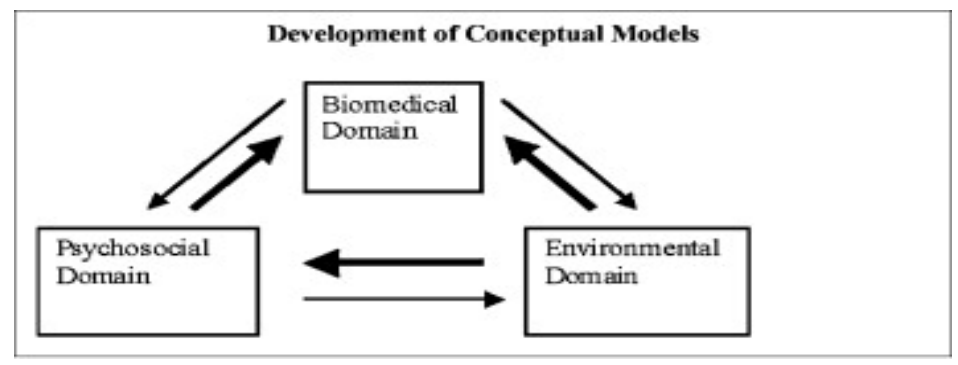

Figure 1: A relationship model of domains for mass-gathering health. (Relative strength of influence is indicated by weights of arrows.) 


\subsection{Environmental domain}

The environmental domain incorporates the geographic features of the venue, whether the event is hosted indoors or outdoors, temperature and humidity and whether the crowd is seated, standing or mobile. Characteristics include crowd attendance, crowd density, crowd dynamics and the availability of drugs and/or alcohol. The environmental domain impacts strongly on the bio-medical domain. Moreover, the environmental domain can also influence the psychosocial domain in regards to how environmental influences impact on crowd behaviour $[9,10]$.

\subsection{Psychosocial domain}

Characteristics of the psychosocial domain include the social influences within a mass gathering such as the examination of individual and crowd behaviour, crowd interests, rationale for attending the event and the use of drugs and alcohol [9].

The characteristics of the psychosocial domain also have a strong influence on the bio-medical domain.

\subsection{Bio-medical domain}

The bio-medical domain includes the health of both the crowd and the individual and also the potential for illness and injury. Characteristics include average age, dominant gender, level of activity and drug and alcohol use. While these indicators are linked to overall risk of illness or injury at the event [9], they have relatively less influence on the overall mass gathering event than the other domains.

Arbon's [9] conceptual framework is unique in the mass gathering literature as its aim is to describe the inter-relationship between the domains of a mass gathering and their elements as outlined in Figure 2.

\section{Investigating the psychosocial domain}

To date, the science of mass gatherings has focused on the environmental and biomedical domains of mass gatherings [11] and there is limited knowledge to support our understanding of the psychosocial domain, including identifiable key features and how these elements interact with each other [12].

An important element of the Arbon model is the potential impact of the psychosocial domain on injury and illness rates due to elements traditionally described as 'crowd mood' and 'crowd type' [12]. Crowd type is a descriptor of the societal sub-culture of a crowd whereas crowd mood is a descriptor of crowd emotion.

Characteristics which unite crowds include sharing close proximity (e.g. being in the mosh pit), similarity (e.g. appearance) and shared interests or cooperative interaction (e.g. love of punk music). Although various categorisations have been developed to describe the collective actions of crowds, Berlonghi's typology [13] of crowd types, outlined in Table 1, is the 
classification system most widely adopted in the literature and is promoted by Emergency Management Australia [2].

\begin{tabular}{|c|c|c|}
\hline Psychosocial domain & Biomedical domain & Environmental domain \\
\hline $\begin{array}{l}\text { Crowd: } \\
\text { a. behavior } \\
\text { b. mood }\end{array}$ & Crowd and individual health status & $\begin{array}{l}\text { Crowd: } \\
\text { a. attendance } \\
\text { b. density }\end{array}$ \\
\hline $\begin{array}{l}\text { Individual: } \\
\text { a. motivation } \\
\text { b. behavior }\end{array}$ & Latent potential for illness and injury & $\begin{array}{l}\text { Venue: } \\
\text { a. bounded/unbounded } \\
\text { b. extendedffocused } \\
\text { c. localefterrain }\end{array}$ \\
\hline$\Leftarrow$ & $\Rightarrow$ & $\Rightarrow$ \\
\hline Crowd interests/morays/ culture & Crowd average age/ gender & Type/ nature of event \\
\hline E & $\Rightarrow$ & $\Rightarrow$ \\
\hline Rationale/ reason for attendance & Activity level (participant/ spectator) & Predominantly seated or mobile \\
\hline$\leftarrow$ & $\Rightarrow$ & $\Rightarrow$ \\
\hline Length of stay & Heat- $\alpha$ cold-related physiology & $\begin{array}{l}\text { Outdoor or indoor weather } \\
\text { (temperature/humidity) }\end{array}$ \\
\hline$\Leftarrow$ & $\Rightarrow$ & $\Rightarrow$ \\
\hline Use of alcohol or drugs & Alcohol- or drug-related physiology & Availability of alcohol $\alpha$ drugs \\
\hline & $v$ & $\Downarrow$ \\
\hline & Effect & Response \\
\hline & Risk of injury or illness: & Level and extent of healthcare services \\
\hline & $\begin{array}{l}\text { a. Patient presentation rate (PPR) } \\
\text { b. Transport to hospital rate (TTHR) }\end{array}$ & \\
\hline
\end{tabular}

Prehosptal and Disaster Medicine $\oplus 2004$ Arbon

Figure 2: $\quad$ Arbon's conceptual model.

Table 1: $\quad$ Crowd types (Berlonghi [13]; EMA [2]).

\begin{tabular}{|l|l|}
\hline \multicolumn{1}{|c|}{ Crowd type } & \multicolumn{1}{c|}{ Comment } \\
\hline Ambulatory & Walking, usually calm. \\
\hline $\begin{array}{l}\text { Disability/limited } \\
\text { movement }\end{array}$ & $\begin{array}{l}\text { Crowd has limited or restricted movement; requires } \\
\text { additional planning. }\end{array}$ \\
\hline Cohesive/spectator & Watching specific activity. \\
\hline Expressive/revelous & Emotional release; for example community fun runs. \\
\hline Participatory & $\begin{array}{l}\text { Involved in actual event; for example, pickets, } \\
\text { marches. }\end{array}$ \\
\hline Aggressive/hostile & Initially verbal, open to lawlessness. \\
\hline Demonstrator & $\begin{array}{l}\text { Organised to some degree; for example, pickets, } \\
\text { marches. }\end{array}$ \\
\hline Escape/trampling & Danger may be real or imaginary. \\
\hline Dense/suffocating & Reduction of individual physical movement. \\
\hline Rushing/looting & $\begin{array}{l}\text { Attempt to acquire/obtain/steal something; for } \\
\text { example, tickets. }\end{array}$ \\
\hline Violent & Attacking/terrorising. \\
\hline
\end{tabular}


Crowd mood, on the other hand, is a descriptor of crowd emotion. The mood of the crowd has been described as an important element in determining crowd behaviour [3] and is associated with a number of factors such as the closeness of people in a crowd, the occurrence of incidents (such as a crowd crush) and/or the effect of the music $[3,14]$. The recognition, tempo or rhythm of songs, the type and attitude of the performers or any combination of these have been found to be closely linked to changes in crowd behaviour [15]. In particular, heavy metal, hardcore punk and rap performances are highly influential on crowd behaviour [15].

Practical strategies to monitor and measure crowd mood and type, along with the resultant behaviour of a crowd, have received limited attention [11]. However, Pines and Maslach [16] developed a matrix that uses the audience profile for assessment of crowd mood. They use descriptors to clearly identify separate groups, such as families, young adults, children, elderly and rival factions and then attach a rating scale (1-5) to these groups. This scale is used to grade the amount of verbal noise, physical movement and overall audience participation as outlined in Table 2 .

Zeitz et al. [11] used the Pines and Maslach model to examine a significant number of mass gathering events and found that crowd mood was an important factor in predicting medical workload at a mass gathering event.

Hutton et al. [17] used a similar methodology to measure crowd behaviour in a systematic way at the 2009 annual schoolies event in South Australia. Crowd behaviour was measured using a simple matrix incorporating Berlonghi's crowd type typology and the Pines and Maslach framework to measure crowd mood as well as additional factors relating to motivation to attend - age, gender, interests, crowd interests/morays/culture, rationale/reason for attendance (if different from motivation to attend) and length of stay.

Hutton et al. [12] adopted this same methodology to examine crowd behaviour at the Adelaide Big Day Out festival in the summer of 2010. In analysing the data, the researchers applied a simple scoring schema to each tool, attributing numerical values to each element to quantify the findings and identify any trends. For the crowd mood descriptors (see Table 2), a score was applied to each element from 1-15. Passive (little or no talking) was assigned a score of 1, passive (little or no physical movement) a score of 2, etc., through to energetic (maybe episodes of violence) being assigned a score of 15. Berlonghi's crowd types (see table 1) were also assigned scores, with ambulatory $=1$, disability/limited movement $=2$, cohesive $=3$ and so on. Finally, a score was attributed to the behaviours observed during the classifications (passive, active, energetic) provided by Pines and Maslach.

The outcomes of these studies suggest that 'crowd type' and 'crowd mood' are measurable indicators of the behaviour of a crowd at an outdoor music festival. In the context of attempting to develop a predictive model of behavioural assessment, it is necessary to identify those factors and processes which act on and transform individual behaviour into the dangerous irrational and high risk behaviour observed at these events. 
Table 2: Crowd mood classification (Pines and Maslach [16]; Zeitz et al. [11]).

\begin{tabular}{|l|l|}
\hline Mood descriptor & Score \\
\hline Passive & 1. Little or no talking \\
\hline & 2. Little or no physical movements \\
\hline & $3 . \quad$ Little or no physical contact \\
\hline Mood descriptor & 4. Little or no audience participation \\
\hline & Score \\
\hline Active & $5 . \quad$ Co-operative \\
\hline & $1 . \quad$ Moderate degree of talking \\
\hline & $2 . \quad$ Moderate degree of physical movements \\
\hline & $3 . \quad$ Moderate degree of physical contact \\
\hline & $4 . \quad$ Moderate degree of audience participation \\
\hline Energetic & $5 . \quad$ Co-operative \\
\hline & $1 . \quad$ Considerable degree of talking \\
\hline & $2 . \quad$ Considerable degree of physical movements \\
\hline & $3 . \quad$ Considerable degree of physical contact \\
\hline & $4 . \quad$ Considerable degree of audience \\
& participation \\
\hline & $5 . \quad$ May be episodes of violence \\
\hline
\end{tabular}

\section{From individual to collective behaviour}

Crowd incidents show different types of group motivation. In some cases there is an interruption of a simple traffic process, such as exiting a stadium or a passenger conveyor, resulting in a critical crowd pressure point. Others fall into the two general behavioural categories of either a flight response or a craze [18]. Flight occurs where people experience either a real or perceived threat. A mass craze is a competitive rush to obtain some highly valued objective.

In an attempt to look at how behaviour might be affected at mass gatherings, Au et al. [19] developed the crowd behaviour model outlined in Figure 3, which demonstrates how the behaviour of individuals combines and interacts with each other to give a collective or group response to a situation.

Deindividuation, group polarisation and groupthink have been identified as the primary processes responsible for converting individual into collective behaviour.

Deindividuation is the feeling of losing one's individuality or personal identity as the result of being submerged in a group and occurs primarily when people are aroused by being in a large group and feel diminished responsibility for their acts.

Group polarisation is the tendency for group involvement to strengthen the average group member's before-involvement inclination. When people discover that others are inclined to feel the same way as they do, they are motivated to shift their opinion further in that direction. 
Eactors Affecting Individual Behaviour

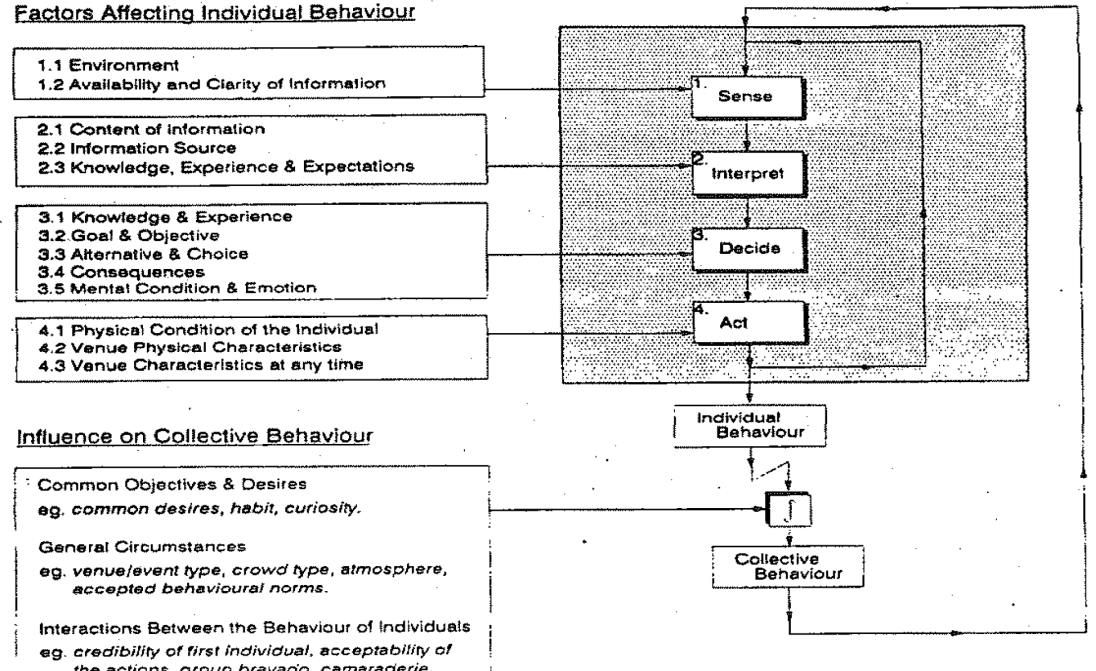

Figure 3: $\quad$ Factors affecting behaviour in public venues (Au et al. [19]).

Groupthink refers to feelings of cohesion within a group. Concern for cohesion leads group members to strive for unanimity, often at the expense of rational decision making. Under conditions of groupthink, people in highly cohesive groups (like cultural subgroups) act as if upsetting the group would be worse than making a wrong decision.

Over the years, sociologists and other scholars have proposed a number of explanations of collective behaviour, which are summarily outlined in Table 3.

Table 3: $\quad$ Snapshot of collective behaviour theories.

\begin{tabular}{|l|l|}
\hline \multicolumn{1}{|c|}{ Theory } & \multicolumn{1}{c|}{ Major assumptions } \\
\hline Contagion theory & $\begin{array}{l}\text { Collective behaviour is emotional and irrational and } \\
\text { results from the hypnotic influence of the crowd. }\end{array}$ \\
\hline Convergence theory & $\begin{array}{l}\text { Crowd behaviour reflects the beliefs and intentions } \\
\text { that individuals already share before they join a } \\
\text { crowd. }\end{array}$ \\
\hline $\begin{array}{l}\text { Emergent norm } \\
\text { theory }\end{array}$ & $\begin{array}{l}\text { People are not sure how to behave when they begin } \\
\text { to interact in collective behaviour. As they discuss } \\
\text { their potential behaviour, norms governing their } \\
\text { behaviour emerge, and social order and rationality } \\
\text { then guide their behaviour. }\end{array}$ \\
\hline Value-added theory & $\begin{array}{l}\text { Collective behaviour results when several conditions } \\
\text { exist, including structural strain, generalized beliefs, } \\
\text { precipitating factors, and lack of social control. }\end{array}$ \\
\hline Social identity theory & $\begin{array}{l}\text { People in a crowd act as one because they share a } \\
\text { common social identity. This identity specifies the } \\
\text { normative behaviour of the group. }\end{array}$ \\
\hline
\end{tabular}




\section{A model outlining elements and processes of the psychosocial domain}

The schema outlined in this paper can now be brought together to outline a preliminary model, outlined in Figure 3, which can provide the basis for developing a methodology and instrument for behavioural assessment at outdoor music festivals.

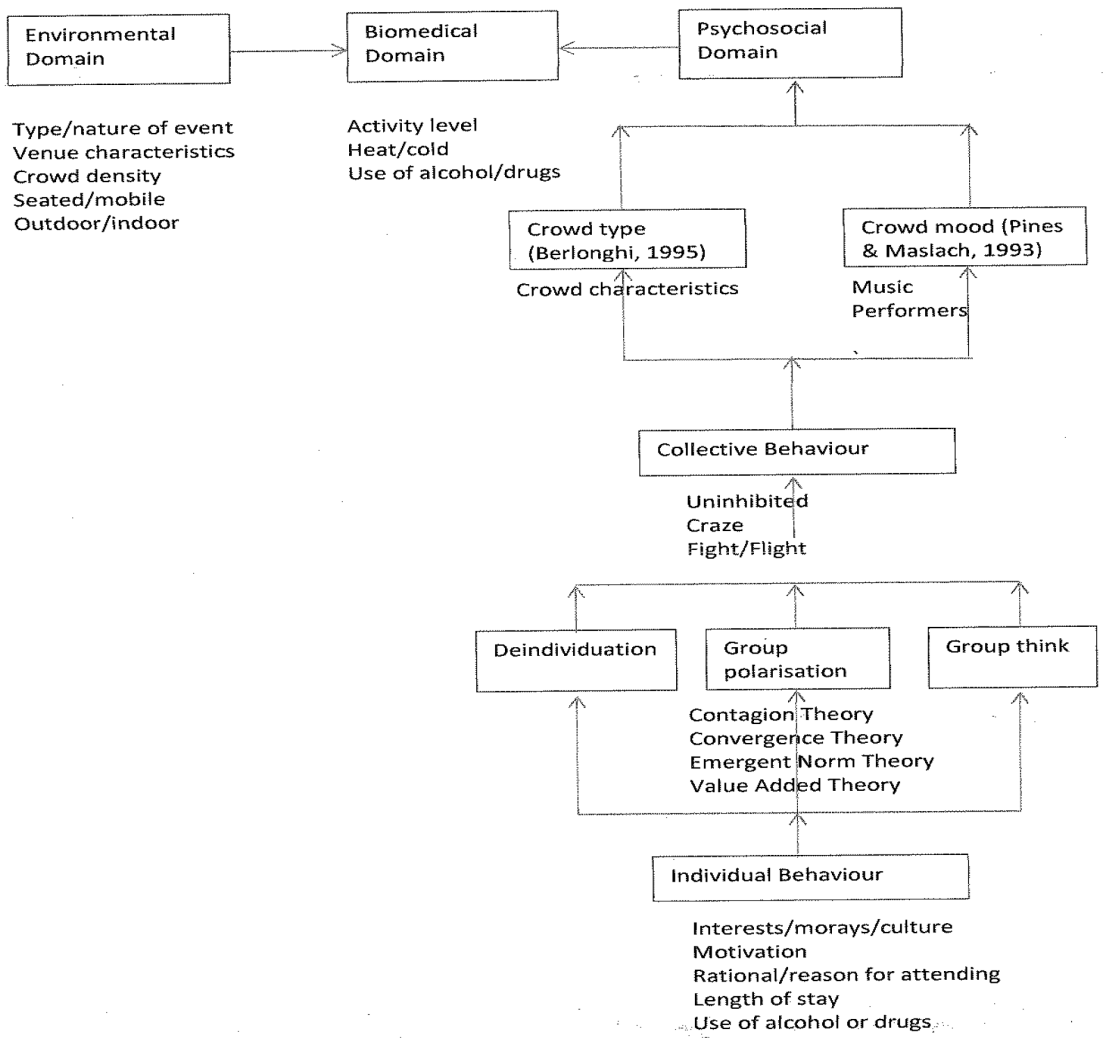

Figure 4: Elements and processes of the psychosocial domain.

\section{Conclusion}

A consideration of the risk factors at outdoor music festivals needs to include behavioural assessment. Characteristics and processes of collective behaviour at these events are usually ignored. The model outlined in this paper clearly highlights factors and processes to be taken into account when developing a risk assessment tool. 


\section{References}

[1] Dickie, J.F., Major crowd catastrophes, Safety Science, 18, pp. 309-320, 1995.

[2] Emergency Management Australia [EMA], Safe and Health Mass Gatherings, Manual 2, Canberra: AGPS, 1999.

[3] Milsten, A., Maguire, B., Bissell, R. and Seaman, K., Mass-gathering medical care: A review of the literature, Prehospital and Disaster Medicine, 17(3), pp. 151-162, 2002.

[4] Sime, J.D., Crowd Psychology and Engineering: Designing for People or Ballbearings? Engineering for Crowd Safety, eds. R.S. Smith and J.F. Dickie, Elsevier Science Publishers: BV, 1993.

[5] Sime, J.D., Crowd psychology and engineering. Safety Science, 21(1), pp. 1-14, 1995.

[6] Upton, M., Risk analysis for major concert events, the benefits of hindsight, Cabinet Office Seminar on Safety at Mass Crowd Events (January 2004), Chicago: Crowdsafe.com Library, 2004.

[7] Upton, M., Safe Event Management, Paper delivered to the Theatre Managers Association Conference, Birmingham, 10 June, 2008.

[8] Tatrai, A., The cost of regret - the role of risk management in event/venue planning (an Australian perspective), Chicago: Crowdsafe.com Library, 2001.

[9] Arbon, P., The development of conceptual models for mass-gathering health, Journal of Prehospital and Disaster Medicine, 19 (3), pp. 208-212, 2004.

[10] Zeitz, K. M., Zeitz, C. J., and Arbon, P., Forecasting medical work at massgathering events: Predictive model versus retrospective review, Prehospital Disaster Medicine, 20 (3), pp. 164-168, 2005.

[11] Zeitz, K., Tan, H.M., Grief, M., Couns, P.C. and Zeitz, C.J. Crowd behaviour at mass gatherings: A literature review, PreHospital and Disaster Medicine, 14 (1), pp. 132-138, 2009.

[12] Hutton, A., Zeitz, K., Brown, S. and Arbon, P., Prehospital and Disaster Medicine, 26 (6), pp. 414-421, 2012.

[13] Berlonghi, A. E., Understanding and planning for different spectator crowds, Safety Science, 18, pp. 239 - 247, 1995.

[14] Earl, C., Parker, E., Tatrai, A. and Capra, M., Influences on crowd behaviour at outdoor music festivals, Environmental Health, 4 (2), pp. 5562, 2004.

[15] Earl, C., Parker, E. and Capra, M., The Management of Crowds and Other Risks at Outdoor Music Festivals: A Review of the Literature, Environmental Health, 5 (1), pp. 37-49, 2005.

[16] Pines, A. and Maslach, C., Experiencing Social Psychology, New York: McGraw-Hill, 1993.

[17] Hutton, A., Munt, R., Zeitz, K., Cusack, L., Kako, M and Arbon, P., Piloting a mass gathering conceptual framework at an Adelaide Schoolies Festival, Collegian, 17, pp. 183-191, 2010. 
88 Safety and Security Engineering V

[18] Fruin, J., Crowd Disasters - A Systems Analysis of Causes and Countermeasures, US National Bureau of Standards pub NBSIR 81-3261, July, 1981.

[19] Au, S., Ryan, M. and Carey, M., Key Principles in Ensuring Crowd Safety in Public Venues, R.A. Smith and J.F. Dickie (ed.) Engineering for Crowd Safety, Amsterdam: Elsevier, 1993. 